\title{
OPTIMIZATION OF DESIGN PARAMETERS OF STEEL CHIMNEYS
}

\author{
Manoj Kr Gupta ${ }^{1}$., Bajpai V.K ${ }^{2}$, Garg T.K. ${ }^{3}$ \\ ${ }^{1}$ Research Scholar, Department of Mechanical Engineering, National Institute of Technology, Kurukshetra \\ ${ }^{2,3}$ Department of Mechanical Engineering, National Institute of Technology, Kurukshetra \\ E-mail: manoj_gupta280@yahoo.com
}

\begin{abstract}
In the highly competitive world of today, it is essential to design an efficient, versatile and cost effective system. To design such systems proper analytical, experimental and numerical tools are needed. Optimum design concepts and methods provide some of the needed tools. When these are properly implemented in to software, these give powerful numerical tools for designing best systems. Formulation of an optimum design problem involves transcribing a verbal description of problem in to a well-defined mathematical statement whose constituent elements are parameters, constraints and objective or merit function. Here, a stack design problem has been developed as a mathematical model. Design variables for the present problem are mean diameters $d i$, shell thickness $t i$, and height $h_{i}$ of ith lift. Design expressions and constraints have been formulated. The intent of the objective function is to minimize total mass and thus, cost of steel chimney to select design vectors [h], [d] and [t] while satisfying the nine constraints. For the present problem Random Search Methods have been used to design flowchart for the optimum solution because these are quite simple to program and are very reliable in finding a nearly optimal solution.
\end{abstract}

Key words: Drag coefficient, Terrain friction drag coefficient, Dynamic lift coefficient, Strouhal number, Frequency of vortex shedding, Natural frequency, Random Search Method.

\section{NOTATION}

$A_{k}$

$\mathrm{Cd}_{d}$ drag coefficient

$\mathrm{C}_{f} \quad$ terrain friction drag coefficient

Cl dynamic lift coefficient

$C_{1} \quad$ Rs cost per unit area of steel sheet

$\mathrm{C}_{2} \quad \mathrm{Rs}$ cost per unit volume of weld

$d_{i} \quad$ mean shell diameter of $i_{\text {th }}$ lift of stack, $m$

D external diameter of stack, $m$

E Modulus of elasticity

$F_{d} \quad$ drag force, $\mathrm{N}$

$\mathrm{Fg}$ gust load, $\mathrm{N}$

$\mathrm{Fl}$ across wind force, $\mathrm{N}$

$f_{n} \quad$ natural frequency of chimney, $\mathrm{Hz}$

$f_{V} \quad$ frequency of shedding pairs of vortices, $\mathrm{Hz}$

g acceleration due to gravity, $\mathrm{m} / \mathrm{sec}^{2}$

$W_{e} \quad$ anticipated weight of ladder and platform, $\mathrm{kg}$

$h_{i} \quad$ height of ith lift of stack, $m$ i lift counter from top to bottom

j grid counter

I area moment of inertia, $\mathrm{mm}^{4}$

M resultant bending moment, $\mathrm{Nm}$

$\mathrm{M}_{\mathrm{E}} \quad$ equivalent end mass, $\mathrm{Kg}$

$\mathrm{Mi}_{\mathrm{i}} \quad$ mass of ith section, $\mathrm{Kg}$

n exponent which depends on surface roughness, number of trial chimneys

P uniformly distributed load, $\mathrm{N} / \mathrm{m}$

$P_{c r} \quad$ critical buckling load, $\mathrm{N}$

St Strouhal number

$t_{i} \quad$ shell thickness of $i_{\text {th }}$ lift of stack, $m$

$\mathrm{U} \quad$ design wind speed at height $\mathrm{Z}, \mathrm{m} / \mathrm{sec}$

$\mathrm{U}_{0}$ basic maximum wind speed at reference height $Z_{0}$

$\bar{U} \quad$ design wind speed averaged over entire stack height, $\mathrm{m} / \mathrm{sec}$

$W_{k} \quad$ total structural weight of stack at $k_{\text {th }}$ section, $\mathrm{kg}$

$z_{i} \quad$ distance measured from base to center of mass of section, $\mathrm{m}$ 
$Z_{k} \quad$ section modulus of $k_{t h}$ section, $\mathrm{m}^{3}$

$\rho_{a}, \rho_{s}$ mass density of air, steel, $\mathrm{kg} / \mathrm{m}^{3}$

$\sigma_{a} \quad$ allowable yield stress of material, $\mathrm{N} / \mathrm{mm}^{2}$

$\Delta \quad$ maximum permissible deflection, $\mathrm{mm}$

$\partial$, y deflection, $\mathrm{mm}$

\section{INTRODUCTION}

Optimization techniques play an important role in structural design, the very purpose of which is to find the best ways so that a designer or a decision maker can derive maximum benefit from the available resources. A stack design problem has been represented as a mathematical model whose constituent elements are parameters, constraints and objective or merit function. Design parameters specify geometry, topology of stack and physical properties of its members. From design parameters, a set of derived parameters are obtained which are defined as behavior constraints e.g., stresses, deflections, natural frequencies and buckling loads etc. The objective or merit function has been formed to minimize total mass and thus, cost of steel chimney (4).

\section{FORMULATION OF DESIGN PROBLEM}

\section{A. Design variables}

For design purpose, self-supported steel chimney is treated as a cantilever beam of variable moment of inertia (1). Chimney is divided into a number of convenient zones as shown in Fig. 1. Design variables for problem are mean diameters $d_{j}$, shell thickness $t_{j}$, and height of $i_{\text {th }}$ lift $h_{i}$. If chimney is divided into $n$ elements, total number of design variables is therefore $3 \times W_{1}, W_{2}, W_{n}$ are structural weights of lifts, $P_{1}, P_{2}, P_{n}$ are uniformly distributed loads on lifts of heights $h_{1}, h_{2}, h_{n}$ and thickness $t_{1}, t_{2}, t_{n}$. Let chimney has an inside diameter $D_{i}$ and physical height $H_{i}$, as determined by technical, operational and pollution considerations.

\section{B. Design Expressions}

For a cantilever chimney, steel structure experiences combined bending moment and shear forces $(6,12 \& 13)$. Wind load over a differential area $\mathrm{dA}$ is shown in Fig 2.

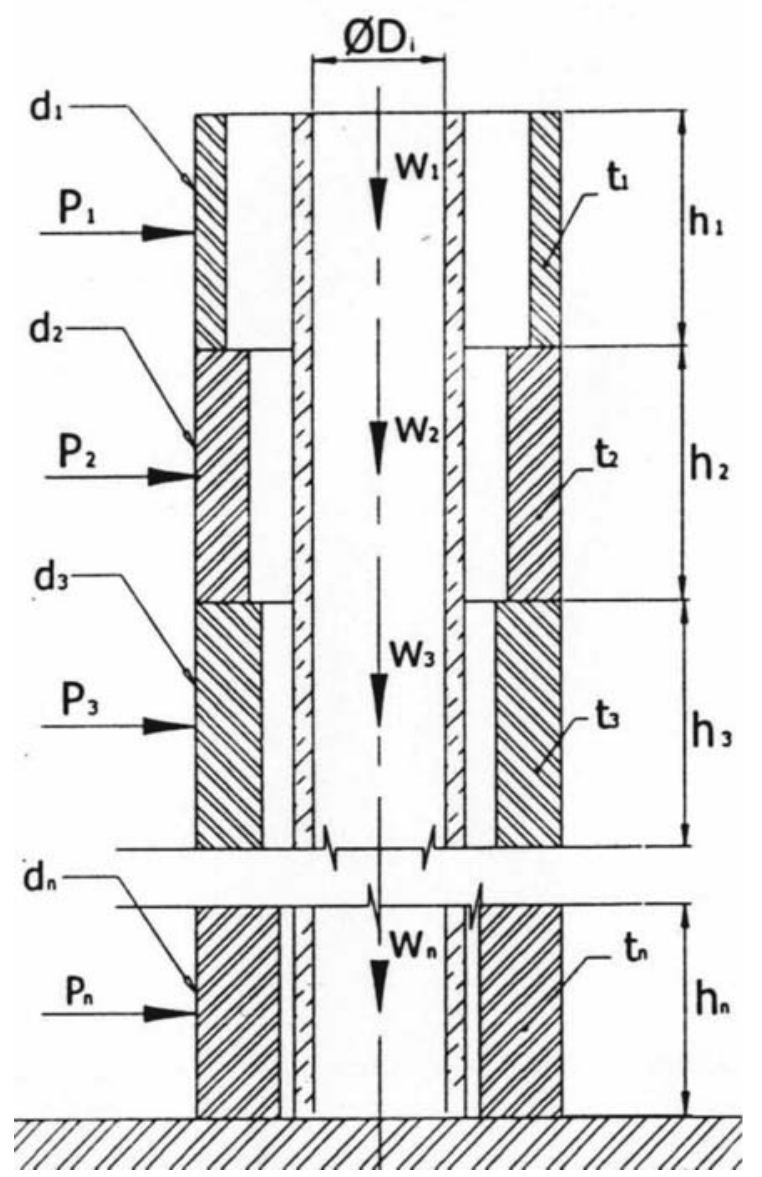

Fig. 1 Chimney Modeled as a Cantilever Beam

Along wind force is composed of static drag force and gust load. Along wind Force $=$ Drag Force + Gust Load

Drag force acting at a distance $z$ measured from base is given by:

$F_{d}=\frac{1}{2} \frac{C_{d} \rho_{a}}{2 n+1}\left(\frac{U_{0}}{Z_{0}^{n}}\right)^{2} D\left(z_{2}^{2 n+1}-Z_{1}^{2 n+1}\right)$

Gust load component acting at any section of stack is given by:

$F_{g}=2.18 U_{0}^{2} C_{d} D \cdot \rho_{a} \sqrt{C_{f}} \frac{1}{Z_{0}^{n}} \frac{1}{n+1}\left(Z_{2}^{n+1}-Z_{1}^{n+1}\right)$

Lift force generated due to vortex shedding is given by:

$F_{1}=\frac{1}{2(2 n+1)} C_{1} \rho_{a}\left(\frac{U_{0}}{Z_{0}^{n}}\right)^{2} D\left(Z_{2}^{2 n+1}-Z_{1}^{2 n+1}\right)$ 
Value of exponent $\mathrm{n}$ is defined in Central Pollution Control Board, Delhi, Probes/ 70/ 1997-98 (10). Values of coefficients $C_{d}, C_{f}$ and $C_{l}$ defined in Manohar (7). Resultant design force which acts at any cross section is vector sum of along wind forces and across wind force:

$$
F=k_{1} \sqrt{\left(F_{d}+F_{g}\right)+r F_{1}}
$$

Where, $r$ is dynamic magnifier at design wind speed and $k_{1}$ is overloading factor which takes into account uncertainties in loading. Resulting bending moment at any cross-section at distance $\mathrm{z}$ from base of chimney due to wind loads is given by:

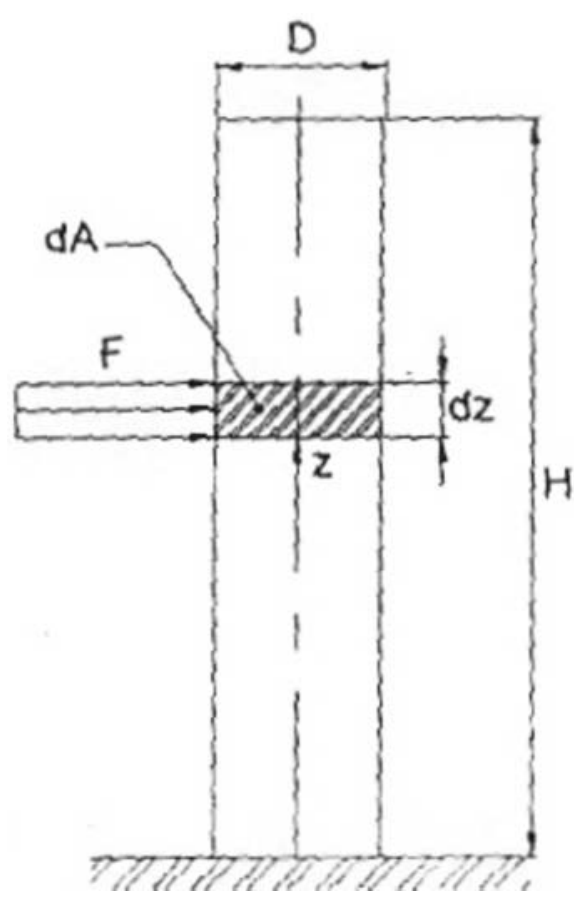

Fig. 2 Wind Load over a Differential Area

$$
M=F \cdot \frac{H-Z}{2}
$$

\section{Formulation of Objective Function}

Cost function: The intent is to minimize total mass and thus, cost of chimney. Total cost of steel chimney depends on:

(a) Area and thickness of steel sheet

(b) Cost of manufacturing (i.e. cost of cutting and welding)

For a cantilever chimney made of $n$ number of lifts of variable thickness, cost function is written as:

$$
\text { Cost }=C_{1} \cdot\left(d_{1} h_{1} t_{1}+d t_{2} h_{2} t_{2}+d_{3} h_{3} t_{3}+\ldots . d_{n} h_{n} t_{n}\right) \pi p,+C_{2}(n-1)
$$

\section{Formulation of Constraints}

Behavior constraint: To prevent material overstressing, resultant of bending and shear stresses and stresses resulting from self-weight of steel structure induced at $k_{\text {th }}$ section is not to be greater than permissible yield stress of material.

$$
\frac{W_{1}}{A_{k}}+\frac{M_{k}}{Z_{k}} \leq \sigma_{a}
$$

Where, $Z_{k}$ is given by:

$$
Z_{k}=\frac{\pi d_{k}^{3} t_{k}}{4 D}
$$

Where, $d_{k}$ and $t_{k}$ are mean shell diameter and thickness at $k_{\text {th }}$ section.

Total structural weight at $k_{t h}$ section is:

$$
W_{1}=\frac{1}{4} \cdot g \cdot p_{s} \cdot \pi \cdot \sum_{i=1}^{k} d_{i} t_{i} h_{i}
$$

Area of section $A_{k}$ is given by:

$$
A_{k}=\pi \cdot d_{k} \cdot t_{k}
$$

For finding moment $M_{k}$, values $z_{2}=H$ and $z_{1}=z_{k}$ are substituted in expressions [2], [3] and [4] and resulting values of $F_{d} F_{g}$ and $F_{l}$ are used in expressions (5) and (6).

Deflection constraint: Deflection under extreme loading conditions must be at most $\Delta$. Therefore, calculated deflection $\partial$ must be less than or equal to $\Delta$. Maximum deflection of a cantilever beam subjected to a uniformly distributed load $P$ as shown in Fig. 3 is given by:

$$
y_{\max }=\frac{1}{8} \cdot \frac{P \cdot H^{3}}{E l}
$$

Expression (12) is used to find top deflection of a self-supported steel chimney by substituting $P=F / H$.

To avoid excessive arithmetic, value of $\mathrm{El}$ is taken as that of base section. It is recommended that 
maximum allowable deflection for a steel chimney is to be less than or equal to $H / 200$ ( 3 \& 5).

$$
\frac{1}{8} \cdot \frac{F \cdot H^{2}}{E I} \leq \frac{H}{200}
$$

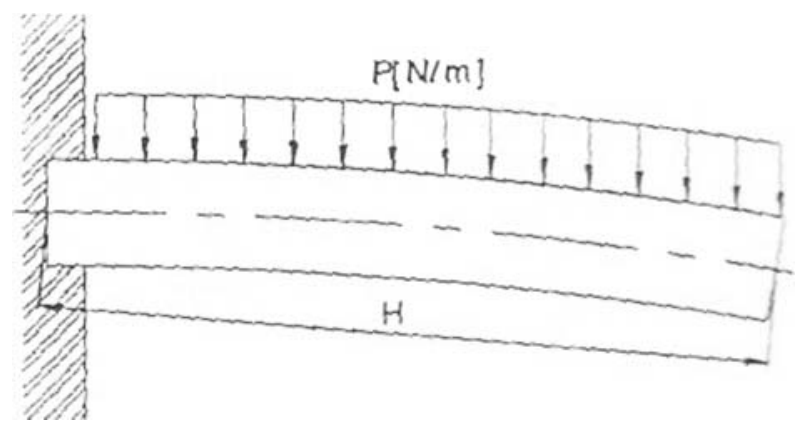

Fig. 3. Deflections due to Wind Loads

Buckling constraint: Stress induced in chimney shell is to be less than buckling stress. Assuming a fixed end condition, critical buckling load is calculated by:

$$
P_{c r}=\frac{\pi^{3} E R_{k}^{3} t_{k}}{4 h_{k}}
$$

Where, $R_{k}$ is radius of stack shell at $k_{t h}$ section, $R_{k}=d_{k} / 2$ and downward self load $W_{k}$ ( for a self-supporting chimney) is to be less than or equal to $P_{c r}$

$$
W_{k} \leq P_{c r}
$$

Constraint on frequency of vortex shedding: Frequency of vortex shedding at design wind speed is given by:

$$
f_{v}=\frac{\bar{U} \cdot S t}{D}
$$

Natural frequency of a cantilever chimney constructed of lifts of different thicknesses is:

$$
f_{n}=\frac{1}{2 \pi} \cdot \sqrt{\frac{3 \cdot E \cdot l}{M_{E} \cdot H^{3}}}
$$

Where, $M_{E}$ is equivalent end mass that would make chimney vibrate at same frequency as does a mass carried at top and is obtained by:

$$
M_{E}=\sum_{i=1}^{n}\left(\frac{Z_{i}}{H}\right)^{2} M_{i}
$$

Resonance is avoided by making natural frequency of chimney as large as possible. This is expressed mathematically as:

$$
f_{n} \geq f_{v}
$$

Diameter constraint: Inner diameter of chimney should not be less than $d_{j}$, as determined by requirements of exit velocity and volume of flue gas to be handled, so

$$
d_{k}-t_{i} \geq d_{i}
$$

Constraints on height of lifts: Maximum height of a lift is often limited to 12.1 meters to account fabrication and installation difficulties, so

$$
h_{i} \leq 12.1
$$

Explicit bounds on thickness of steel sheet: To avoid practical difficulties while manufacturing, restrictions on minimum and maximum thickness of structural steel sheet are:

$$
\begin{gathered}
t_{\min } \leq t_{i} \leq t_{\max } \\
h_{\min } \leq h_{i}
\end{gathered}
$$

Minimum cost and mass design problem is to select design vectors (h), (d) and (t) to minimize cost function expression (7), while satisfying the mlnine constraints of Ex. (8) through Ex. (23)

\section{E. Standard Definition of problem}

After normalizing constraints, using defined data, optimum formulation for design of industrial steel chimney is obtained. 


$$
\text { Find } X=\left|\begin{array}{c}
d_{1} \\
d s u b \\
d_{3} \\
\cdots \\
t_{1} \\
t_{2} \\
t_{3} \\
\cdots \\
h_{1} \\
h_{2} \\
h_{3}
\end{array}\right|
$$

Method (2, 8, 14 \& 15). Random search methods are very simple to program and are quite reliable in finding a nearly optimal solution. Flowchart developed based on this method is shown in Figures 4,5 and 6. Steps involved are as below:

(i) Generation of a trial design vector using one random number for each design variable.

(ii) Verification of constraints at trial design vector. Eqality constraints are considered satisfied whenever their magnitudes lie within a specified tolerance. If any constraint is violated, continue generating a new trial vector that satisfies all constraints.

$f(x)=C_{1} \cdot\left(d_{1} h_{1} t_{1}+d_{2} h_{2} t_{2}+d_{3} h_{3} t_{3}+\ldots d_{n} h_{n} t_{n}\right) \pi p_{s}+C_{2}(n-1)$

Subject to behavior constraint:

$$
\frac{W_{k}}{A_{k}}+\frac{M_{k}}{Z_{k}}-\sigma_{a} \leq 0
$$

Deflection constraint:

$$
F \cdot H-0.04 \cdot E \cdot I \leq 0
$$

Buckling constraint:

$$
-\frac{1}{4} \cdot g \cdot p_{x} \cdot \pi \cdot \sum_{i=1}^{k} d_{i} t_{i} h_{i}+\frac{\pi^{3} \cdot E \cdot R_{k}^{3} \cdot t_{k}}{4 h_{k}} \leq 0
$$

Vortex shedding frequency:

$$
-\frac{1}{2 \pi} \sqrt{\frac{3 \cot E \cdot I}{\sum_{i=1}^{n}\left(\frac{Z_{i}}{H}\right)^{3} M_{i} H^{3}}}+\frac{\bar{U} \cdot S t}{D} \leq 0
$$

Inner diameter constraint:

$$
-d_{k}+t_{k}+d_{i} \leq 0
$$

Height of lifts constraint:

$$
h_{i}-12.1 \leq 0
$$

Thickness constraint:

$$
t_{\min } \leq t_{j} \leq t_{\max }
$$

\section{OPTIMUM SOLUTION TECHNIQUES}

There are many techniques to solve problem defined in expressions (24) to (32). One of these methods for present problem is Random Search (iii) If all constraints are satisfied, retain current trial vector as best design if it gives a reduced objective function value compared to previous best available design. Otherwise discard current

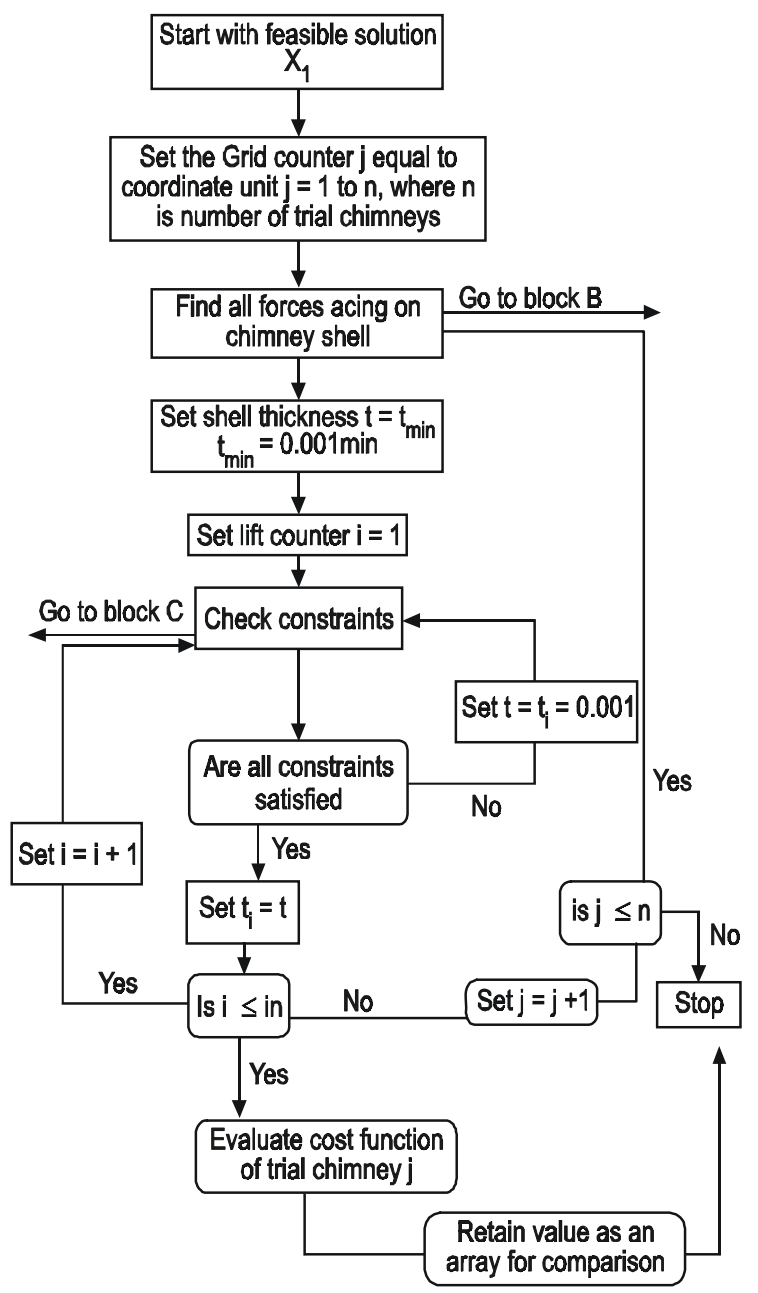

Fig. 4. Flow Chart of the Main Program 


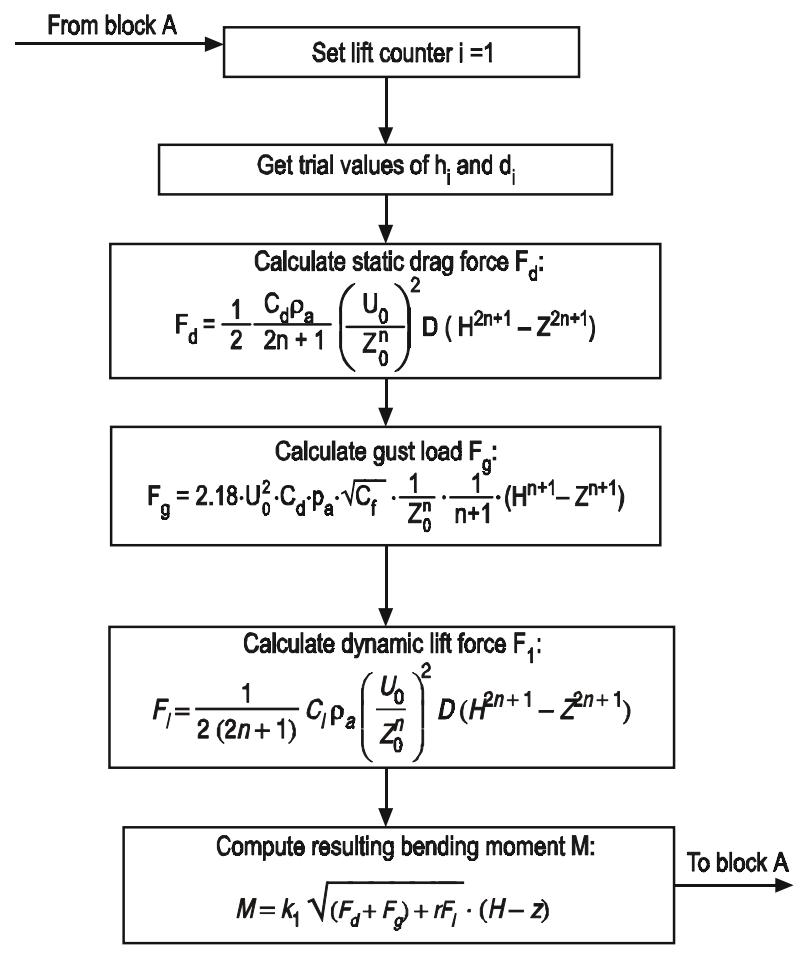

Fig. 5. Block B of Fig. 4

feasible trial vector and proceed to step 1 to generate a new trial design vector.

(iv) Best design available at the end of generating a specified maximum number of trial design vectors is taken as solution of the constrained optimization problem.

\section{CONCLUSIONS}

For designing a structural system, conventional and optimum design processes are used at different stages of design. In conventional design, it would be sometimes difficult to decide whether to increase or decrease the size of a particular structural element to satisfy the constraints. Furthermore, conventional design can lead to uneconomical design and involve a lot of calendar time. These shortcomings have been omitted by applying optimization technique. For this, design variables, expressions and constraints have been formulated. The objective or merit function has been formed to minimize total mass and thus, cost of steel chimney expression [7] to select design vectors [h], [d] and [t], while satisfying the nine constraints of expressions [8] through [23] with the help of random search methods.

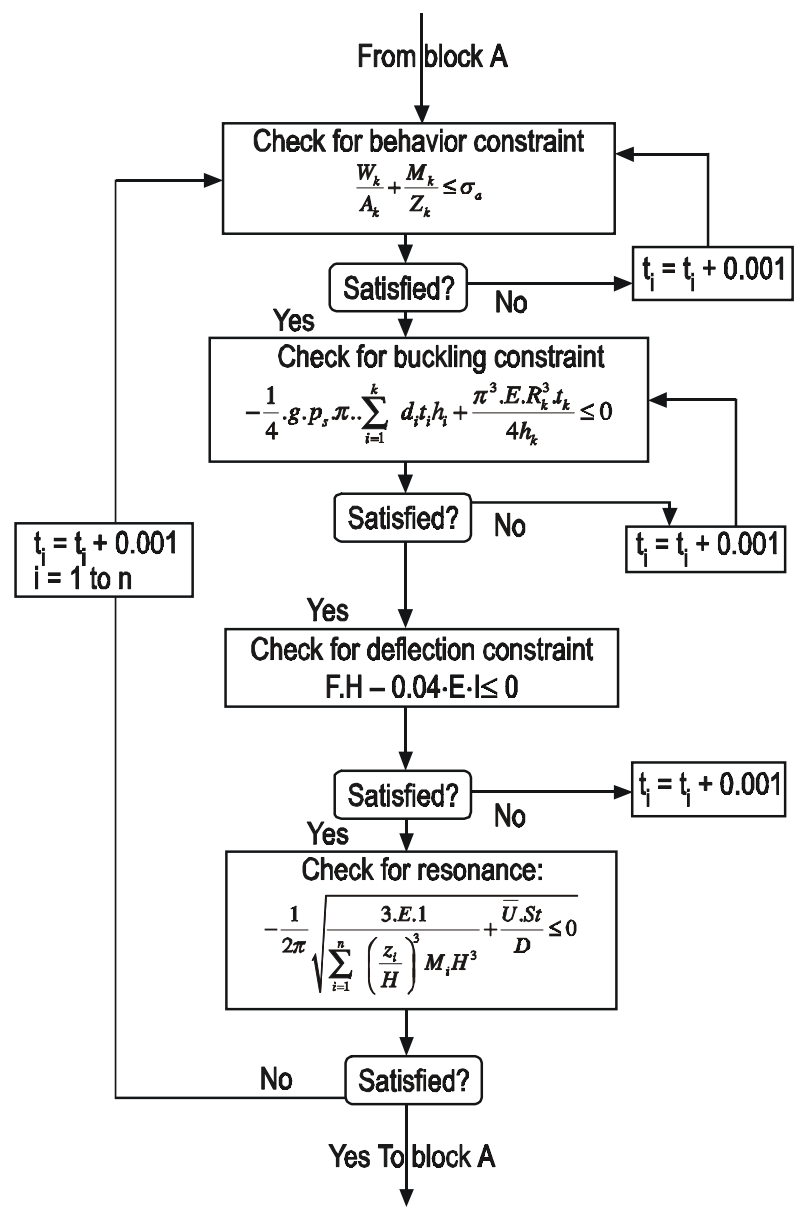

Fig. 6. Block $\mathrm{C}$ of Fig. 4

\section{REFERENCES}

[1] Agarwal, S. K. and Lakshmy, P., 1994, Computer Analysis of Tall Free Standing Chimney, Journal of The Institution of Engineers (India), 74, pp. 187-196.

[2] Seetharaman, S., 1998, Knowledge Based Systems for Offshore Engineering Applications, Journal of The Institution of Engineers (India), 79, pp. 35-40.

[3] Bolton, A., March 1994, Wind Excitation of Steel Chimneys, The Structural Engineer, 72 (5), pp. 75-80.

[4] lyengar, N. G. R., Optimization in Structural Design, pp. 41-48.

[5] 2003 IS: 6533-1989 (Part II), Code of Practice for Design and Construction of Steel Chimney, Structural Aspect, Bureau of Indian Standards, pp. 1-20.

[6] Kaemmer, Klaus K., et al., 2005, eThe CICIND Chimney Book. f Comite International Des Cheminees Industrielles (CICIND), pp. 99-114.

[7] Manohar, S. N., 1985, eTall Chimney Design and Construction f, Tata McGraw-Hill, New Delhi, pp. 29-40. 
[8] Rao, S. S., 1999, eEngineering Optimization: Theory and Practice f, New Age Publishers, New Delhi, pp. 432-433.

[9] Gilles Oudin, 2005, Optimization of Steel Stacks, CICIND.

[10] 97-98 eAssessment of Impact to Air Environment: Guidelines for Conducting Air Quality Modeling f, Central Pollution Control Board, Delhi, Probes/ 70.

[11] Arora, Jasbir S., 2007, eOptimization of Structural and Mechanical Systems f, World Scientific, pp. 261.

[12] Dyrbye Claes and Hansen Svend Ole, 1999, eWind Loads on Structures f, John Wiley \& Sons, pp. 49-137.

[13] Solari Giovanni, 2002, The Role of Analytical Methods for Evaluating the Wind-Induced Response of Structures, Journal of Wind Engineering and Industrial Aerodynamics, 90 (12-15), pp. 1453-1477.

[14] Lange and Kenneth, 2006, eOptimization f, Springer-Verlag, New York.
[15] Baba, N., 1981, Convergence of a Random Optimization Method for Constrained Optimization Problems, Journal of Optimization Theory and Applications, 33 (4), pp. 451-461.

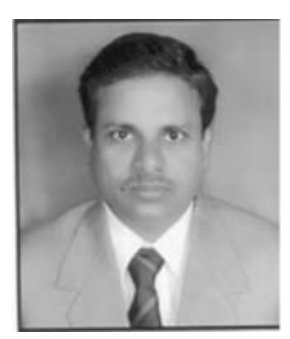

Manoj $\mathrm{Kr}$ Gupta is a research Scholar at the Department of Mechanical Engineering, National Institute of Technology, Kurukshetra. He is a graduate in Mechanical Engineering from Regional Engineering College, Kurukshetra and post graduate in Design from National Institute of Technology, Kurukshetra. He has 8 years of industrial experience. He is an expert in Steel Chimneys, Vibration Engineering and Machine Design. $\mathrm{He}$ is having more than 15 research papers in International \& National Journals and Conferences to his credit. 